\title{
Direct detection experiments at the neutrino dipole portal frontier
}

\author{
Ian M. Shoemaker ${ }^{1,2}$ and Jason Wyenberg ${ }^{1}$ \\ ${ }^{1}$ Department of Physics, University of South Dakota, Vermillion, South Dakota 57069, USA \\ ${ }^{2}$ Center for Neutrino Physics, Department of Physics, Virginia Tech, Blacksburg, Virginia 24061, USA
}

(Received 5 December 2018; published 10 April 2019)

\begin{abstract}
Heavy sterile neutrinos are typically invoked to accommodate the observed neutrino masses, by positing a new Yukawa term connecting these new states to the neutrinos in the electroweak doublet. However, given our ignorance of the neutrino sector, we should explore additional interactions such sterile neutrinos may have with the Standard Model. In this paper, we study the dimension-5 operator which couples the heavy state to a light neutrino and the photon. We find that the recent XENON1T direct detection data can improve the limits on this "neutrino dipole portal" by up to an order of magnitude over previous bounds for tau neutrinos. Future direct detection experiments may be able to extend bounds for all three neutrino flavors down to the level probed by SN1987A.
\end{abstract}

DOI: $10.1103 /$ PhysRevD.99.075010

\section{INTRODUCTION}

The fact that neutrinos are massive is one of the key observational facts indicating that the Standard Model (SM) of particle physics is incomplete. Most models of neutrino masses posit new right-handed states, which are singlets under the SM gauge groups. These neutral fermion singlets have been predominantly studied in connection with neutrino masses via the neutrino portal interaction, $\mathcal{L} \supset N H L$, where $N$ is the singlet fermion, $L$ is the $\mathrm{SM}$ lepton doublet, and $H$ is the Higgs doublet. For this reason, singlet fermions can play the role of a "sterile" neutrino (i.e., uncharged under the electroweak symmetry), and they mix with the left-handed neutrinos after the Higgs acquires a vacuum expectation value. For a review of existing bounds on sterile neutrinos, see Ref. [1].

However, the standard neutrino portal interaction may not be the predominant interaction these states have with the SM. They may also interact via a "neutrino dipole portal" interaction, which after electroweak symmetry breaking can be written as

$$
\mathcal{L}_{\mathrm{NDP}} \supset d\left(\bar{\nu}_{L} \sigma_{\mu \nu} F^{\mu \nu} N\right)+\text { H.c., }
$$

where $F_{\mu \nu}$ is the electromagnetic field strength, $\sigma_{\rho \sigma}=$ $\frac{i}{2}\left[\gamma_{\rho}, \gamma_{\sigma}\right], \nu_{L}$ is the SM neutrino, and the coefficient $d$ with units of (mass) $)^{-1}$ controls the strength of the interaction. This interaction has been considered in the context of the

Published by the American Physical Society under the terms of the Creative Commons Attribution 4.0 International license. Further distribution of this work must maintain attribution to the author(s) and the published article's title, journal citation, and DOI. Funded by SCOAP ${ }^{3}$.
MiniBooNE events [2-8] and has also been studied in the context of IceCube data [9] and at the upcoming SHiP experiment [10]. A summary of existing constraints can be found in Refs. [9,10].

In this paper, we will study the neutrino dipole portal (NDP) at the XENON1T direct detection experiment using their approximately 1 ton-year exposure [11]. Despite not finding evidence of dark matter (DM) scattering, XENON1T is nearly at the level where it can start seeing events from solar neutrinos. Many prior works have used neutrinos at direction detection experiments to study various beyond SM neutrino interactions [12-23]. At the few kilo-electron-volt recoil energies of XENON1T, the Boron-8 (B8) neutrinos make the largest contribution, comprising approximately 0.02 background events in the 1 ton-year sample [11]. However, if neutrinos have additional interactions beyond electroweak forces, this rate could be larger and already detectable. To get an approximate idea of the sensitivity to the NDP, we can compare the SM cross section, $d \sigma / d E_{R} \simeq G_{F}^{2} Q_{w}^{2} m_{N} / 4 \pi$, with the NDP cross section, $d \sigma / d E_{R} \simeq d^{2} \alpha Z^{2} / E_{R}$.

Thus, to achieve approximately 1 event at XENON1T, we would very roughly expect

$$
d \simeq \sqrt{50 \frac{G_{F}^{2} Q_{w}^{2} m_{N} E_{R}}{4 \pi \alpha Z^{2}}} \sim 10^{-6} \mathrm{GeV}^{-1} \sqrt{\frac{E_{R}}{\mathrm{keV}}}
$$

where the factor of 50 comes from needing a 50-fold increase in the SM cross section for the "neutrino floor" to presently be detectable. We expect the estimate in Eq. (2) to be valid up to singlet fermion masses of order Boron-8 energies, $m_{4} \sim E_{\nu} \sim 10 \mathrm{MeV}$. Although the above estimates are simplistic, they provide us with ample motivation to carry out a more complete analysis. Indeed, a dipole 


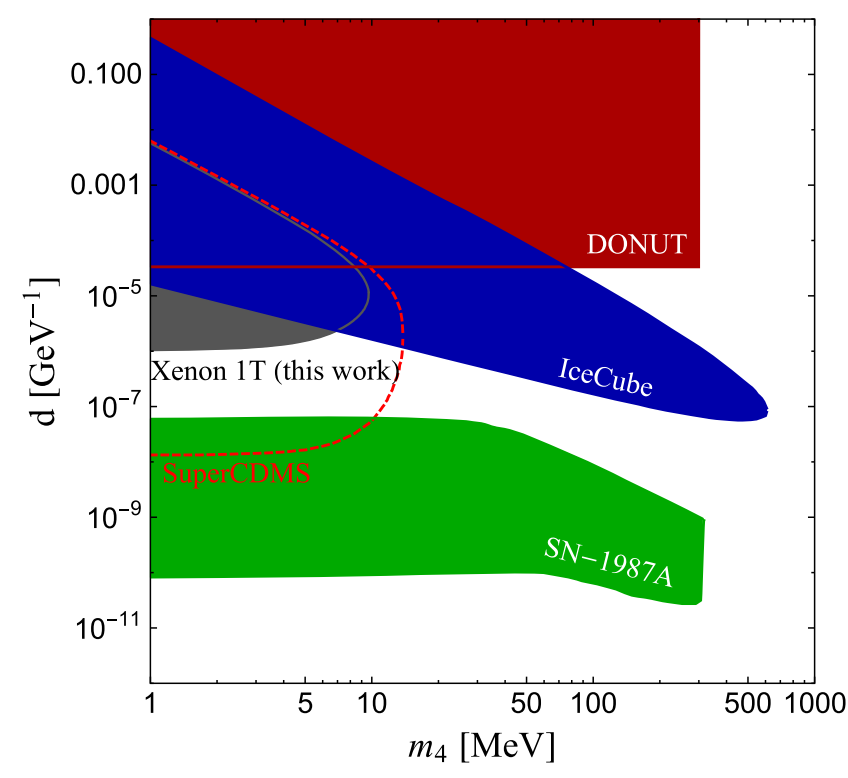

FIG. 1. These are the expected sensitivity curves for the tauflavored NDP based on the XENON1T data [11] and a future SuperCDMS exposure (dashed curve for projection). The relevant previously published exclusion limits of SN1987A [10], IceCube [9], and DONUT [9,24] are also shown.

strength at the $d \simeq 10^{-6} \mathrm{GeV}^{-1}$ level is competitive with a variety of known constraints on the NDP $[7,9,10]$. We summarize our main findings, which demonstrate that XENON1T already provides the leading constraints on coupling to tau flavor neutrinos up to $10 \mathrm{MeV}$ masses, in Fig. 1. The constraints provided by XENON1T on electron and muon flavor neutrinos are not novel as they are by constraints from CHARM-II, MiniBooNE, and LSND [10]. Future high-exposure/low-threshold direct detection can improve the bounds down to the SN1987A region.

We highlight that the bounds we derive are only weakly dependent on the assumed lepton-flavor structure in Eq. (1). However, existing constraints on this NDP interaction are strongly dependent on the assumed flavor dependence of the operator. In particular, muon- (Fig. 4) and especially tau-flavored (Fig. 1) interactions are sufficiently weakly constrained that the new bounds from direct detection experiments provide new sensitivity. The bounds on electron flavored NDP are already sufficiently strong from existing data (see Ref. [10]) that the direct detection bounds considered here do not probe new parameter space, and we therefore omit them in what follows.

The remainder of this paper is organized as follows. In Sec. II, we compute a realistic spectrum of nuclear recoil events at XENON1T from solar neutrinos mediated by the NDP interaction. We find there that the new state will typically decay outside the detector after it is produced and that an incoming neutrino is unlikely to undergo much up-scattering in the Earth prior to arrival at the detector. In Sec. III, we look at what improvements can come in the near term, focusing on a future run of SuperCDMS.
Then, in Sec. IV, we discuss the nature of models giving rise to the NDP, along with potential ways in which future work could refine and extend the analysis carried out here.

\section{NEUTRINO TRANSITION MAGNETIC MOMENT RATES}

\section{A. Mechanism}

Incoming solar neutrinos may up-scatter to a heavy state $N$ through the NDP operator via the process shown in Fig. 2. The coherent cross section for neutrino-nucleus scattering via a NDP reads

$$
\begin{aligned}
\frac{d \sigma_{\nu n \rightarrow N n}}{d E_{R}}= & d^{2} \alpha Z^{2} F^{2}\left(E_{R}\right)\left[\frac{1}{E_{R}}-\frac{m_{4}^{2}}{2 E_{\nu} E_{R} m_{N}}\left(1-\frac{E_{R}}{2 E_{\nu}}+\frac{m_{N}}{2 E_{\nu}}\right)\right. \\
& \left.-\frac{1}{E_{\nu}}+\frac{m_{4}^{4}\left(E_{R}-m_{N}\right)}{8 E_{\nu}^{2} E_{R}^{2} m_{N}^{2}}\right],
\end{aligned}
$$

where $Z$ is the atomic number, $E_{R}$ is the nuclear recoil energy, $E_{\nu}$ is the incident neutrino energy, $m_{N}$ is the mass of the target nucleus, $m_{4}$ is the mass of the heavy sterile neutrino, and $F\left(E_{R}\right)$ is the nuclear form factor.

\section{B. Event rates in Xenon1T detector}

Neutrino scattering at dark matter direct detection experiments has been widely studied [17,25-34] and depending on the range of energies may include contributions from solar, atmospheric, and the diffuse supernova background. In fact, the original DM direct detection proposal from Goodman and Witten [35] was an extension of the detection method for solar and reactor neutrinos via neutral currents by Drukier and Stodolsky [36].

The nuclear recoil spectrum of neutrino induced scattering events can be computed via

$$
\frac{d R^{\alpha}}{d E_{R}}=M T \times \int_{E_{\nu}^{\min }} \frac{d \Phi_{\nu}^{\alpha}}{d E_{\nu}} \frac{d \sigma_{\nu n \rightarrow N n}^{\alpha}}{d E_{R}}\left(E_{\nu}, E_{R}\right) d E_{\nu},
$$

where $\alpha=e, \mu, \tau$ indexes the neutrino flavor, $M T$ is the exposure of the XENON1T experiment, and $\Phi_{\nu}$ is the solar neutrino flux. The ${ }^{8} B$ solar neutrino reaction creates electron

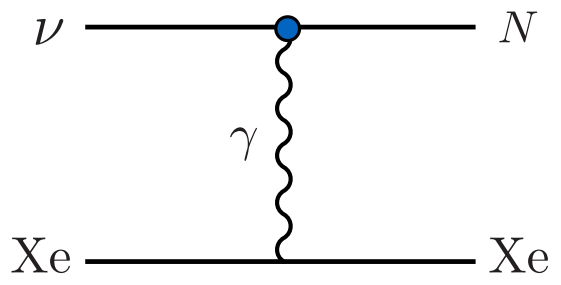

FIG. 2. The neutrino dipole portal $\mathcal{L}_{\mathrm{NDP}} \supset d\left(\bar{\nu}_{L} \sigma_{\mu \nu} F^{\mu \nu} N\right)$ allows for a neutrino to up-scatter off a nucleus to a heavy neutral lepton state $N$. This produces a distinctive recoil spectrum, while the newly produced heavy neutral lepton decays outside the detector. 


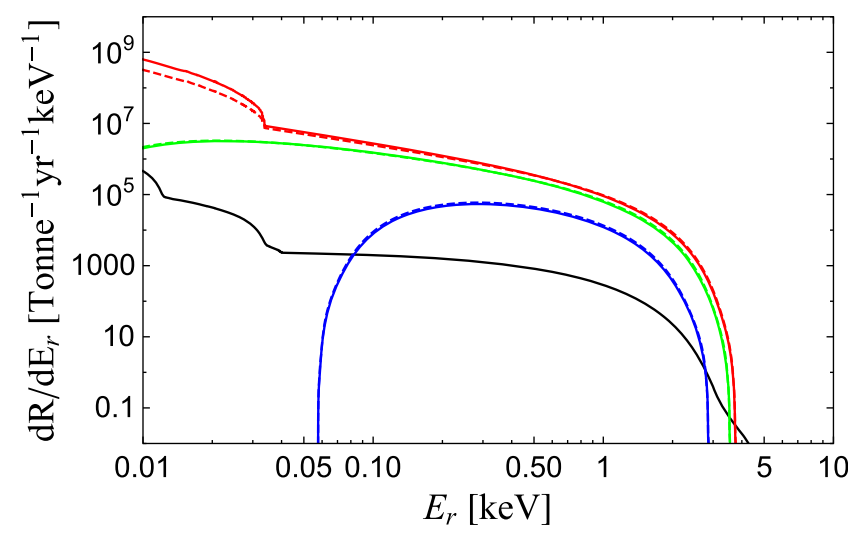

FIG. 3. Event rates of nuclear scattering via a NDP in the Xenon $1 \mathrm{~T}$ detector with $d=10^{-6} \mathrm{GeV}^{-1}$ for masses $m_{4}=1 \mathrm{MeV}$ (red), $m_{4}=5 \mathrm{MeV}$ (green), and $m_{4}=10 \mathrm{MeV}$ (blue). Dashed (solid) curves represent scattering with tau/muon (electron) neutrinos. Also shown is the standard model $\nu$-nucleus scattering rate (black curve).

neutrinos which oscillate into muon and tau flavor states before reaching Earth. For concreteness, we employ the electron neutrino survival probability profile from Ref. [37] to estimate flux rates for the three flavor states of solar neutrinos.

The function $E_{\nu}^{\min }\left(E_{R}\right)$ is the minimum energy of the incident neutrino to up-scatter to the state of mass $m_{4}$ while producing a nuclear recoil $E_{R}$ :

$$
E_{\nu}^{\min }\left(E_{R}\right)=\frac{m_{4}^{2}+2 m_{N} E_{R}}{2\left[\sqrt{E_{R}\left(E_{R}+2 m_{N}\right)}-E_{R}\right]} .
$$

The solar neutrino ${ }^{8} B$ flux provides the dominant contribution to NDP nuclear scattering in the XENON1T experiment. We normalize the ${ }^{8} B$ spectrum to the flux $\phi_{8_{B}}=5.1 \times 10^{6} \mathrm{~cm}^{2} \mathrm{~s}^{-1}$ [38]. For illustration, in Fig. 3, we plot the expected recoil spectrum for a range of possible $\nu_{4}$ masses. As one would expect, at low masses, there is little dependence on the recoil spectrum, while at masses around approximately $10 \mathrm{MeV}$, the rate starts to get very suppressed.

A detected nuclear recoil event will create a signal of $n$ photoelectrons (PEs) given by a Poisson distribution with expectation value $\bar{n}$, given by

$$
\bar{n}=E_{R} L_{y}\left(E_{R}\right) g_{1} .
$$

$L_{y}\left(E_{R}\right)$ is the light yield as a function of $E_{R}$ as shown in Ref. [39], and detector photon gain is $g_{1}=0.144 \pm 0.007$ [40]. The event rate is then given by

$$
\frac{d R^{\alpha}}{d n}=\int \operatorname{Eff}\left[E_{R}\right] \frac{d R^{\alpha}}{d E_{R}} \times \operatorname{Poiss}(n \mid \bar{n}) d E_{R},
$$

where $\operatorname{Eff}\left[E_{R}\right]$, the efficiency as a function of nuclear recoil energy, is given in Fig. 1 of Ref. [11]. Finally, we model the total signal rate as

$$
\frac{d R^{\alpha}}{d S 1}=\sum_{n=1}^{\infty} \operatorname{Gauss}(S 1 \mid n, \sqrt{n} 0.5) \times \frac{d R^{\alpha}}{d n},
$$

with the 0.5 factor coming from the uncertainty of the $1 \mathrm{PE}$ bin size. We find that the range of applicable $S 1$ values is $3<S 1<20$, and we neglect signals outside of this range for our analysis.

\section{Exclusion curves}

The result of the XENON1T experiment excludes a portion of the $m_{4}, d$ neutrino dipole portal parameter space. Following a Bayesian approach, with a signal $s$ and background $b$, an upper limit on $s$ can be determined as

$$
s_{u p}=\frac{1}{2} F_{\chi^{2}}^{-1}[p, 2(n+1)]-b,
$$

where $F_{\chi^{2}}^{-1}$ is the inverse cumulative $\chi^{2}$ distribution and $n$ is the number of observed events such that $2(n+1)$ is the number of degrees of freedom. The $p$ factor is given by the expression

$$
p=1-\alpha\left(F_{\chi^{2}}[2 b, 2(n+1)]\right),
$$

where $\alpha$ is $1-C L$ and $C L$ is the confident limit [41]. An alternative statistical analysis may employ the Likelihood Profile method [42] incorporating the binned energy data. A check of the calculated exclusion curve for $d$ for several values of $m_{4}$ showed nearly identical results between a rudimentary likelihood profile method and the $\chi^{2}$ approach employed here.

For the XENON1T data of the $0.9 \mathrm{t}$ reference mass, with two observed events and an expected background of 1.34 events (the lower range of 1.62 events with 0.28 uncertainty), $s_{u p}=6.53$. Figure 1 shows the $90 \%$ confidence exclusion in the $\left(m_{4}, d\right)$ plane. Also shown are excluded regions from previously published results (see the caption for details). For reference, we also show the current and future direct detection sensitivity to the muon-flavored NDP in Fig. 4.

\section{Up-scattering and decay considerations}

Notice that in principle the dipole interaction admits the possibility of $\nu \rightarrow N$ up-scattering prior to the neutrino flux arriving at the detector. At minimum, a neutrino traverses approximately $1 \mathrm{~km}$ to reach the underground detector. We will find that the process of up-scattering in the Earth is irrelevant for the parameters of interest. The total cross section for up-scattering is roughly estimated as [10]

$$
\sigma_{\nu \rightarrow N} \simeq \alpha Z^{2}|d|^{2} \times \log \left(\frac{4 E_{\nu}^{2}}{m_{4}^{4} R_{\text {nuc }}^{2}}\right) .
$$

For an incoming solar neutrino with Boron-8 energies $E_{\nu} \simeq$ $10 \mathrm{MeV}$ while traversing a distance of $1 \mathrm{~km}$ through the Earth, we find that $d$ would need to be 


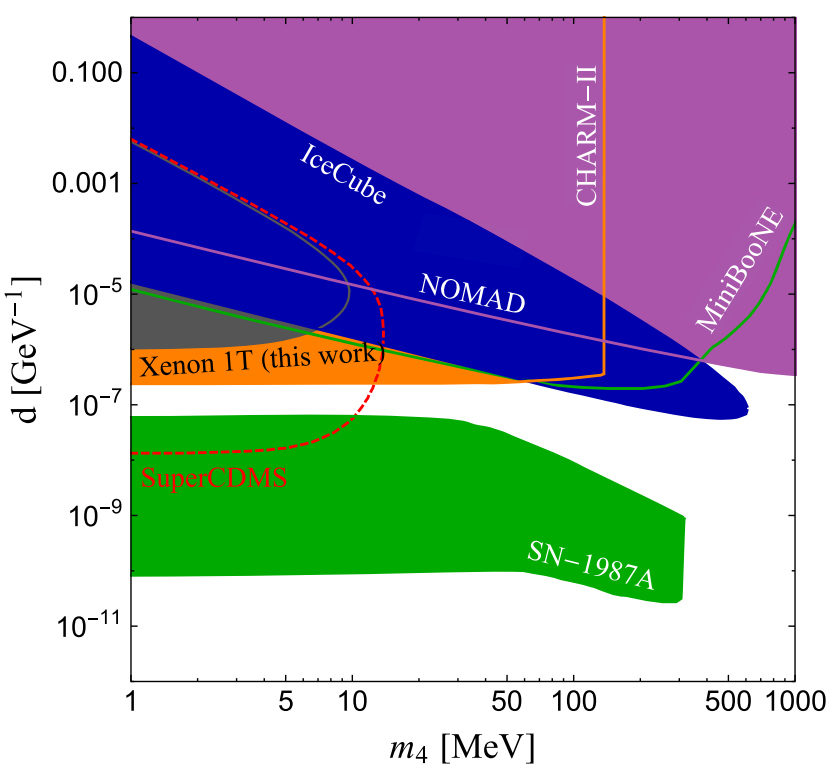

FIG. 4. Expected sensitivity to muon-flavored NDP at XENON1T and a future SuperCDMS exposure (dashed curve for projection). Included are bounds from NOMAD [9,43], CHARM [9,44], MiniBooNE [10,45], IceCube [9], and SN1987A [10].

$$
\begin{aligned}
d & \simeq \sqrt{\frac{1}{(1 \mathrm{~km}) n_{\oplus} \alpha Z^{2} \log \left(\frac{4 E_{\nu}^{2}}{m_{4}^{4} R_{\mathrm{nuc}}^{2}}\right)}} \\
& \simeq 0.14 \mathrm{GeV}^{-1},
\end{aligned}
$$

where we assumed that the dominant contribution to the terrestrial density is silicon. Dipole strengths this strong are already excluded by a number of independent probes including DONUT and IceCube ( $\nu_{\tau}$ transitions) [9] and by CHARM-II, MiniBooNE, and LSND [10] ( $\nu_{\mu}$ transitions).

Shortly after being produced through up-scattering in the detector, the $\nu_{4}$ state will eventually decay. If this decay, $\nu_{4} \rightarrow \nu+\gamma$, happens inside the detector volume, the resultant photon could potentially cause the signal to be thrown away as background. Of course, if the initial nuclear energy deposition in the up-scattering $\nu+\mathrm{Xe} \rightarrow \nu_{4}+\mathrm{Xe}$ is sufficiently far from the final decay of the $\nu_{4}$, one may still be able to perform a search using either only the nuclear recoil events or a dedicated search aimed at the spatially/ temporally correlated nuclear and photon signals unique to the NDP model. Such an analysis is beyond the scope of this work but could result in bounds stronger than the analysis performed here.

To ensure that the nuclear recoil events we model have not been vetoed by the collaboration, we only exclude regions of parameter space where the newly produced $\nu_{4}$ state decays outside of the detector. To do this, we impose a penalty factor on the neutrino dipole portal scattering rates to account for the possibility that the heavy state decays in the detector shortly after being produced. This penalty factor takes the form

$$
P_{\mathrm{D}}=e^{-l_{\mathrm{det}} / \ell_{D}}
$$

where $l_{\text {det }}$ is a characteristic length scale of the detector, which we take to be $100 \mathrm{~cm}$, and the boosted decay length for a $\nu_{4}$ with energy $E_{4}$ is

$$
\ell_{D} \equiv \gamma \beta \tau \simeq \frac{16 \pi E_{4}}{d^{2} m_{4}^{4}} \sqrt{\left(\frac{E_{4}}{m_{4}}\right)^{2}-1}
$$

The above penalty factor was inserted into the integrand of Eq. (4), using $E_{4}=E_{\nu}-E_{R}$. This penalty factor determines the upper boundary of our exclusion curves in Figs. 1 and 4. For the decay $N \rightarrow \nu+\gamma$ to occur inside within $100 \mathrm{~cm}$, we would need $d \gtrsim 4 \times 10^{-4} \mathrm{GeV}^{-1}$ for $E_{4} \simeq 10 \mathrm{MeV}$ and $m_{4}=5 \mathrm{MeV}$.

\section{FUTURE DIRECT BOUNDS: SUPERCDMS ULTRALOW THRESHOLDS}

The SuperCDMS Collaboration [46] anticipates the detection of low-energy nuclear recoil events which could exclude values of $d$ several orders of magnitude lower than the Xenon 1T data. For an order of magnitude estimate, a 1 ton-year exposure of the SuperCDMS experiment was modeled. Using the published efficiency curve of the SuperCDMS experiment [47] (see Fig. 4 in Ref. [47]), the estimated background from solar neutrino nuclear recoils is approximately 460 events. For a $3 \sigma$ discovery signal above the statistical uncertainty, approximately 65 signal events are required. Figure 1 includes a projected exclusion curve of the SuperCDMS experiment.

Worth noting is the fact that the SuperCDMS Collaboration may soon be probing the neutrino floor as solar neutrino backgrounds become prominent in their data. For exposures much higher than 1 ton-year, the detector sensitivity plateaus and cannot probe any lower values of $d$. This is shown in Fig. 5 as the neutrino background systematic uncertainties (dominated by the ${ }^{8} B$ flux uncertainty of

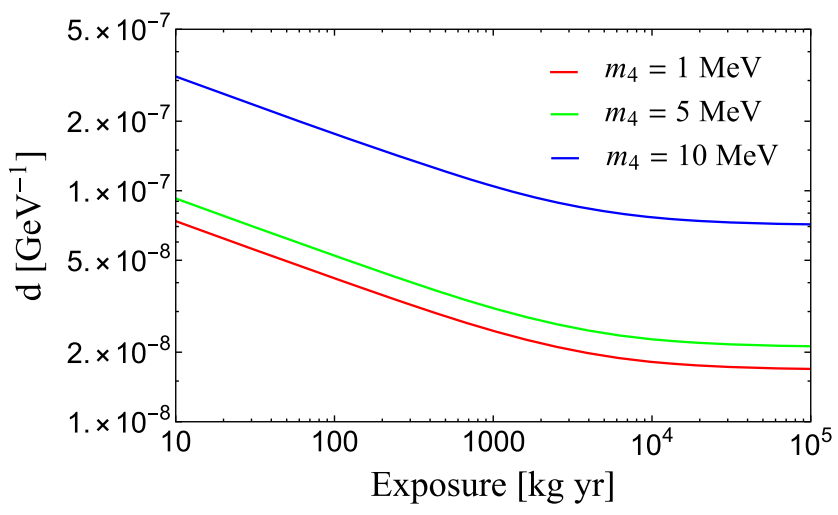

FIG. 5. Anticipated exclusion reach of the SuperCDMS experiment as a function of exposure, eventually plateauing due to systematic uncertainties. 
$\left.0.2 \times 10^{6} \mathrm{~cm}^{2} \mathrm{~s}^{-1}[38]\right)$ obscure a potential signal from the neutrino magnetic moment.

Eventually, $p p$ solar neutrinos may contribute to the neutrino background at direct detection experiments. This would require very low-energy thresholds and would result in a large enhancement of the event rate. For the mechanism of the neutrino transition magnetic moment discussed in this paper, we estimate that a future detector would require a recoil energy threshold below approximately $1 \mathrm{eV}$ to observe the $p p$ flux, and only if $m_{4}$ is below approximately $100 \mathrm{keV}$.

The upper limit of $m_{4}$ that could be excluded by the Xenon $1 \mathrm{~T}$ experiment is approximately $10 \mathrm{MeV}$ due to the maximum incoming energy of the relevant solar neutrino flux. For the exposure of the Xenon 1T data, this maximum energy is that of the solar neutrino B8 flux. To probe higher values of $m_{4}$, the atmospheric neutrino flux could be employed, but this requires an exposure of approximately $10^{7}$ ton-years for a Xenon experiment.

\section{MODEL DISCUSSION AND CONSISTENCY WITH COSMOLOGY}

Throughout the paper, we have assumed that the NDP term, $\mathcal{L}_{\mathrm{NDP}} \supset d\left(\bar{\nu}_{L} \sigma_{\mu \nu} F^{\mu \nu} N\right)$, dominates the phenomenology of the heavy singlet lepton $N$ but have not commented on the implications of such an operator. As discussed in Ref. [10], if $N$ has a large Majorana mass, the NDP interaction can generate a large active neutrino mass. However, this concern is mitigated if the Dirac mass is large compared to the Majorana mass. Moreover, it has been known that the Zee model [48] of neutrino masses can naturally accommodate large magnetic moments [49]. Other models that have been shown to allow for large magnetic moments consistently are the Barr-Freire-Zee spin-suppression mechanism [50] and horizontal flavor symmetries [51] (see also Ref. [52]).

Bounds additional to those considered here can be derived from cosmology. In particular, the NDP interaction can delay the decoupling of neutrinos from the thermal bath in the early Universe [9]. In the standard scenario, neutrinos decouple around temperatures $\sim \mathrm{MeV}$, and departures from this may negatively impact observations sensitive to the radiation energy density. We roughly estimate that the rate of neutron-photon collisions induced by NDP scales as $\Gamma_{\nu \gamma} \simeq d^{2} T^{7} / m_{N}^{2}$. By requiring that this be below the Hubble rate $T^{2} / m_{\mathrm{Pl}}$ at mega-electron-volt temperatures, we find that $d \lesssim 10^{-4} \sqrt{m_{N} / \mathrm{GeV}}$. Given that this is weak compared to the other bounds we show in the figures, we ignore it.

\section{CONCLUSION}

We have shown that the recent XENON1T data can be used to constrain the neutrino dipole portal for tau flavour states at new levels of sensitivity. The solar neutrinos which are nearly detectable at direct detection experiments would already have been observed if the NDP interaction were sufficiently large. Future improvements in searches such as these will come from large scale, low-threshold experiments which will be capable of seeing large numbers of solar neutrino events.

We note that, although we have focused on nuclear recoil events here, one could extend the analysis to include electron recoil events. Given the reduction in the c.m. energy from scattering on electrons, this method will only allow sensitivity to sterile masses $m_{4} \lesssim 0.5 \mathrm{MeV}$.

Lastly, we note that our computation of the excluded NDP strengths has been intentionally conservative. This is largely due to the imposition of the penalty factor, Eq. (13), which vetoes events in which the singlet fermion decay $\nu_{4} \rightarrow \nu+\gamma$ occurs within the detector volume and may therefore complicate discrimination from $e / \gamma$ backgrounds since it yields a nonstandard $S 1$ and $S 2$ signal in XENON1T. A similar effect arising from DM-induced nuclear excitations which redecay to photon final states on short time scales was studied in Ref. [53]. A future analysis may be able to conduct a dedicated search for the nonstandard $S 1$ and $S 2$ signals associated with the NDP.

\section{ACKNOWLEDGMENTS}

We are very grateful to Laura Baudis and Dongming Mei for helpful discussions. This work in supported by the U.S. Department of Energy under the Award No. DESC0019163.
[1] A. Atre, T. Han, S. Pascoli, and B. Zhang, J. High Energy Phys. 05 (2009) 030.

[2] S. N. Gninenko, Phys. Rev. Lett. 103, 241802 (2009).

[3] S. N. Gninenko, Phys. Rev. D 83, 015015 (2011).

[4] D. McKeen and M. Pospelov, Phys. Rev. D 82, 113018 (2010).

[5] M. Masip and P. Masjuan, Phys. Rev. D 83, 091301 (2011).
[6] S. N. Gninenko, Phys. Lett. B 710, 86 (2012).

[7] M. Masip, P. Masjuan, and D. Meloni, J. High Energy Phys. 01 (2013) 106.

[8] E. Bertuzzo, S. Jana, P. A. N. Machado, and R. Z. Funchal, Phys. Rev. Lett. 121, 241801 (2018).

[9] P. Coloma, P. A. N. Machado, I. Martinez-Soler, and I. M. Shoemaker, Phys. Rev. Lett. 119, 201804 (2017). 
[10] G. Magill, R. Plestid, M. Pospelov, and Y.-D. Tsai, Phys. Rev. D 98, 115015 (2018).

[11] E. Aprile et al. (XENON Collaboration), Phys. Rev. Lett. 121, 111302 (2018).

[12] M. Pospelov, Phys. Rev. D 84, 085008 (2011).

[13] R. Harnik, J. Kopp, and P. A. N. Machado, J. Cosmol. Astropart. Phys. 07 (2012) 026.

[14] M. Pospelov and J. Pradler, Phys. Rev. D 85, 113016 (2012); 88, 039904(E) (2013).

[15] M. Pospelov and J. Pradler, Phys. Rev. D 89, 055012 (2014).

[16] P. Coloma, P. Huber, and J. M. Link, J. High Energy Phys. 11 (2014) 042.

[17] D. G. Cerdeo, M. Fairbairn, T. Jubb, P. A. N. Machado, A. C. Vincent, and C. Bhm, J. High Energy Phys. 05 (2016) 118; 09 (2016) 048(E).

[18] J. B. Dent, B. Dutta, S. Liao, J. L. Newstead, L. E. Strigari, and J. W. Walker, Phys. Rev. D 96, 095007 (2017).

[19] E. Bertuzzo, F. F. Deppisch, S. Kulkarni, Y. F. P. Gonzalez, and R. Z. Funchal, J. High Energy Phys. 04 (2017) 073.

[20] B. Dutta, S. Liao, L. E. Strigari, and J. W. Walker, Phys. Lett. B 773, 242 (2017).

[21] D. A. Sierra, N. Rojas, and M. H. G. Tytgat, J. High Energy Phys. 03 (2018) 197.

[22] M. C. Gonzalez-Garcia, M. Maltoni, Y. F. Perez-Gonzalez, and R. Z. Funchal, J. High Energy Phys. 07 (2018) 019.

[23] C. Bœhm, D. G. Cerdeño, P. A. N. Machado, A. O.-D. Campo, and E. Reid, J. Cosmol. Astropart. Phys. 01 (2019) 043.

[24] R. Schwienhorst et al. (DONUT Collaboration), Phys. Lett. B 513, 23 (2001).

[25] B. Cabrera, L. M. Krauss, and F. Wilczek, Phys. Rev. Lett. 55, 25 (1985).

[26] J. Monroe and P. Fisher, Phys. Rev. D 76, 033007 (2007).

[27] L. E. Strigari, New J. Phys. 11, 105011 (2009).

[28] A. Gutlein et al., Astropart. Phys. 34, 90 (2010).

[29] J. Billard, L. Strigari, and E. Figueroa-Feliciano, Phys. Rev. D 89, 023524 (2014).

[30] F. Ruppin, J. Billard, E. Figueroa-Feliciano, and L. Strigari, Phys. Rev. D 90, 083510 (2014).

[31] J. B. Dent, B. Dutta, J. L. Newstead, and L. E. Strigari, Phys. Rev. D 93, 075018 (2016).
[32] C. A. O’Hare, Phys. Rev. D 94, 063527 (2016).

[33] R. Essig, M. Sholapurkar, and T.-T. Yu, Phys. Rev. D 97, 095029 (2018).

[34] J. Wyenberg and I. M. Shoemaker, Phys. Rev. D 97, 115026 (2018).

[35] M. W. Goodman and E. Witten, Phys. Rev. D 31, 3059 (1985).

[36] A. Drukier and L. Stodolsky, Phys. Rev. D 30, 2295 (1984); 30, 2295 (1984).

[37] B. Aharmim et al. (SNO Collaboration), Phys. Rev. C 88, 025501 (2013).

[38] K. Abe et al. (Super-Kamiokande Collaboration), Phys. Rev. D 83, 052010 (2011).

[39] D. S. Akerib et al. (LUX Collaboration), arXiv:1608.05381.

[40] E. Aprile et al. (XENON Collaboration), Phys. Rev. Lett. 119, 181301 (2017).

[41] M. Tanabashi et al. (Particle Data Group), Phys. Rev. D 98, 030001 (2018).

[42] E. Aprile et al. (XENON100 Collaboration), Phys. Rev. D 84, 052003 (2011).

[43] J. Altegoer et al. (NOMAD Collaboration), Nucl. Instrum. Methods Phys. Res., Sect. A 404, 96 (1998).

[44] D. Geiregat et al. (CHARM-II Collaboration), Phys. Lett. B 232, 539 (1989).

[45] A. A. Aguilar-Arevalo et al. (MiniBooNE Collaboration), Phys. Rev. Lett. 98, 231801 (2007).

[46] R. Agnese et al. (SuperCDMS Collaboration), Phys. Rev. Lett. 121, 051301 (2018).

[47] R. Agnese et al. (SuperCDMS Collaboration), Phys. Rev. D 97, 022002 (2018).

[48] A. Zee, Phys. Lett. 93B, 389 (1980).

[49] K. S. Babu, D. Chang, W.-Y. Keung, and I. Phillips, Phys. Rev. D 46, 2268 (1992).

[50] S. M. Barr, E. M. Freire, and A. Zee, Phys. Rev. Lett. 65, 2626 (1990).

[51] R. Barbieri and R. N. Mohapatra, Phys. Lett. B 218, 225 (1989).

[52] M. Lindner, B. Radovi, and J. Welter, J. High Energy Phys. 07 (2017) 139.

[53] C. McCabe, J. Cosmol. Astropart. Phys. 05 (2016) 033. 